\title{
Prediction of 5-Fluorouracil Toxicity Associated With Dihydropyrimidine Dehydrogenase Gene (DPYD) Polymorphism Using the Secondary Structure Prediction Programs
}

\section{Kyonghee Son*}

Department of Cell and Developmental Biology, University of Massachusetts Medical School, Worcester, MA 01655, USA

\begin{abstract}
5-fluorouracil (5-FU) is rapidly degraded by dihydropyrimidine dehydrogenase (DPD), the first and rate-limiting enzyme in the catabolic pathway of 5-FU and pyrimidines. A tolerable therapeutic dose of 5-FU for a DPD-normal patient can make a DPD-deficient patient intolerable. These inter-individual variations in the body's response and tolerance to drugs can be attributed to dihydropyrimidine dehydrogenase gene (DPYD) single nucleotide polymorphisms (SNPs), among others. To save a patient's life and money, health professionals need a convenient and reliable way to find out a patient's tolerance to 5-FU prior to clinical trials or 5-FU therapy. Here I present a simple, easy and fast way to predict an individual's intolerance to 5-FU using the secondary structure prediction programs, YASPIN, PSIPRED and Jpred 3, freely available to anyone. These programs predict the DPD secondary structure with and without mutation (s) within DPYD so that an impact of the mutation-induced structural changes on functional sites of human DPD domains can be deduced. Among 11 SNPs analyzed as samples, two missense mutations, D949V (SNP A2846T) and C953S (SNP G2858C), in the DPD domain V are predicted to cause disruption of the domain core responsible for [4Fe-4S] clusters. Furthermore, a point mutation in a splicing region (In $14 \mathrm{G} 1 \mathrm{~A}$ ) in DPYD is predicted to produce truncated DPD mRNAs (exon 14 skipping) and disabled DPD proteins (missing 55 amino acids from D581 to N635) which cause a complete loss of DPD activity. SWISS-MODEL predicts significant change in the three dimensional structures of human DPD in the presence of exon 14 skipping, D949V and C953S mutation. Thus, prediction by these secondary structure prediction programs provides useful and reliable information about toxicity associated 5-FU due to mutation (s) in DPYD.
\end{abstract}

Keywords: Single nucleotide polymorphisms; Genotype; DPD activity; Efficacy; DPD functional domains; Three dimensional structure

\section{Abbreviation}

DPD: Dihydropyrimidine Dehydrogenase Enzyme; SNPs: Single Nucleotide Polymorphisms; aa: Amino Acid

\section{Introduction}

Some patients benefit from a standardized therapy but others do not because our body's response to drugs varies with an individual's genetic makeup. In the postgenomic era, patients are better off with customized therapies based on their own genotype for effective responses and acceptable tolerance to a drug of choice.

5 -fluorouracil (5-FU) is an analog of the pyrimidine uracil which acts as a pyrimidine antagonist active against colorectal, urinary bladder, breast, prostate and ovarian cancer. Its cytotoxicity can be due to 5-FU anabolites, fluoropyrimidine nucleotides. Fluorodeoxyuridine monophosphate competes with uracil to bind with thymidylate synthetase, interfering with thymidine synthesis and subsequently DNA synthesis and repair. Fluorodeoxyuridine triphosphate is incorporated into DNA and interferes with DNA synthesis. Fluorouridine 5-triphosphate is incorporated into RNA in place of UTP, interfering with RNA processing and synthesis. It is commonly administered by daily, weekly, and monthly bolus injection, or continuous infusion. Many dosing schedules exist and vary with disease state, a patient's genetic make-up, sex, age, route of administration, and ongoing treatment. With its relatively short half-life, most of parenterally administered 5-FU is rapidly degraded in most patients by dihydropyrimidine dehydrogenase (DPD), the first and rate-limiting enzyme in the catabolic pathway of 5-FU and pyrimidines. This requires higher doses to obtain therapeutic response at the expense of intolerable adverse effects such as leucopenia, mucositis, diarrhea, nausea, vomiting, hand-foot syndrome, and cognitive impairment $[1,2]$. These toxic effects also apply to cancer patients receiving capecitabine, an orally-administered prodrug that is enzymatically converted to $5-\mathrm{FU}$ in the body.

An identical therapeutic dose of 5-FU which a DPD-normal patient is tolerable to or able to cope with can make a DPD-deficient patient intolerable or not able to continue taking because of adverse side effects. The benefits of treatment over side effects may be granted in the case of a life-threatening situation with no other drugs are available or effective. These inter-individual variations in the body's response and tolerance to drugs can be attributed to single nucleotide polymorphisms (SNPs), among others. Human DPD gene (DPYD, NC_000001) is localized to chromosome 1 p21.3 and is translated into 1025 amino acids $(\mathrm{MW}=\sim$ $114 \mathrm{KDa})$. In fact, there was a small population of patients with DPYD mutations who exhibited severe side effects following 5-FU therapy [2]. In addition, patients showing 5-FU toxicity (the toxicity group) had more abnormal DPYD genotypes than patients with no toxicity

${ }^{*}$ Corresponding author: Kyonghee Son, Department of Cell and Developmenta Biology, University of Massachusetts Medical School, 55 Lake Avenue North Worcester, MA 01655, USA, Tel: 508-856-8659; Fax: 508-856-1033; E-mail: KyongH.Son@umassmed.edu

Received November 13, 2015; Accepted December 10, 2015; Published December 16, 2015

Citation: Son K (2015) Prediction of 5-Fluorouracil Toxicity Associated With Dihydropyrimidine Dehydrogenase Gene (DPYD) Polymorphism Using the Secondary Structure Prediction Programs. J Pharmacogenomics Pharmacoproteomics 6: 154. doi:10.4172/2153-0645.1000154

Copyright: (c) 2015 Son K. This is an open-access article distributed under the terms of the Creative Commons Attribution License, which permits unrestricted use, distribution, and reproduction in any medium, provided the original author and source are credited. 
Citation: Son K (2015) Prediction of 5-Fluorouracil Toxicity Associated With Dihydropyrimidine Dehydrogenase Gene (DPYD) Polymorphism Using the Secondary Structure Prediction Programs. J Pharmacogenomics Pharmacoproteomics 6: 154. doi:10.4172/2153-0645.1000154

Page 2 of 6

(the control group) [3]. As expected, the pharmacokinetics of 5-FU in a DPD-deficient patient changes: as 5-FU metabolism slows down, its half-life increases with a decrease in its clearance [4].

The purpose of this paper is to predict changes in the secondary structure of DPD domains due to missense point mutations in the DPYD using the secondary structure prediction programs and to deduce whether the mutation-induced structural changes disrupt the functional sites of DPD domains involved in 5-FU metabolism. Thirteen DPYD SNPs were reported from patients who underwent 5-FU monotherapy and showed severe toxicity but two of them were synonymous mutations which has nothing to do with DPD (Table 1). Therefore, eleven nonsynonymous SNPs were chosen and analyzed for the predictability of those programs.

\section{Method}

Amino acid sequence alignment, protein structure prediction and protein isoelectric point (pI) estimation. The amino acid sequence of DPD in phylogenetically related fourteen eukaryotes and the secondary structure elements of DPD domains are aligned using the Clustal W program. The protein sequences used for alignment correspond to the following accession numbers: Sus scrofa (pig: NP_999209.1); Homo sapiens (human: NP_000101.2); Pan troglodytes (chimpanzee: XP_513583.2 predicted); Mus musculus (mouse: NP_740748.1); Rattus norvegicus (rat: NP_112289.1); Bos taurus (cattle:NP_776466.1); Canis familiaris (dog: XP_537061.2 predcited); Gallus (chicken: XP_426639.2 predicted); Danio rerio (zebrafish: NP_998058.1); Caenorhabditis elegans (worm: NP_508927.2); Drosophila melanogaster (fruit fly: NP_572538.1); Anopheles gambiae (mosquito: XP_309214.2); Arabidopsis thaliana (thale cress: NP_188408.1); Oryza sativa (rice: NP_001048053.1). The pIs of each domain with and without SNP are calculated using Emboss [5]. Human DPD monomer is divided into five domains with their distinct functions for 5-FU breakdown according to pig liver DPD monomer as they share 92\% homology. The secondary structure prediction programs, YASPIN [6], PSIPRED [7], and Jpred 3 [8], are used to predict the secondary structure of DPD domains with and without SNP. The predicted structures of human DPD are compared with the crystallographic structure of pig liver DPD as a reference [9]. A three dimensional (3D) structure modeling program, SWISS-MODEL, is used to predict pig and human DPD domains with and without a point mutation [10].

Toxicity prediction. Toxicity is predicted by utilizing the DPD structure-function relationship described below.

1. Get the DPYD and DPD sequence from the NCBI website

2. Identify point mutation by comparing and analyzing the DPYD DNA sequence and DPD protein sequence to find an amino acid substitution corresponding to a base substitution

3. Go to the secondary prediction program websites (YASPIN, PSIPRED, and Jpred3)

\begin{tabular}{|c|c|c|c|c|c|c|c|}
\hline Domain & Polypeptide $^{a} /$ Nucleotide $^{b}$ & Function ${ }^{c}$ & SNPd & Exon ${ }^{e}$ & Allele & aa change ${ }^{f}$ & $\mathrm{pl}^{\mathrm{g}}$ \\
\hline 1 & $\begin{array}{l}27-173(147 \text { aa }) / \\
79-519(441 \mathrm{bp} \text {, exon } 2-6)\end{array}$ & N-terminal Fe-S clusters & $\begin{array}{l}\text { None } \\
\text { T85C } \\
\text { A496G }\end{array}$ & $\begin{array}{l}2 \\
6\end{array}$ & ${ }^{*} 9 \mathrm{~A}$ & $\begin{array}{l}\text { C29R } \\
\text { M166V }\end{array}$ & $\begin{array}{l}8.20 \\
8.42 \\
8.20\end{array}$ \\
\hline 2 & $\begin{array}{l}174-286(113 \mathrm{aa}) / \\
520-858(339 \mathrm{bp} \text {, exon } 6-9) \\
\& \\
442-524(83 \mathrm{aa}) / \\
1324-1572(249 \mathrm{bp}, \text { exon } 11-13)\end{array}$ & FAD binding & $\begin{array}{l}\text { None } \\
\text { G623A } \\
\text { A775G } \\
\text { T1391C }\end{array}$ & $\begin{array}{l}6 \\
8 \\
12\end{array}$ & $\begin{array}{l}\text { New } \\
\text { New } \\
\text { New }\end{array}$ & $\begin{array}{l}\text { R208Q } \\
\text { K259E } \\
\text { V464A }\end{array}$ & $\begin{array}{l}4.85 \\
4.70 \\
4.61 \\
4.85\end{array}$ \\
\hline 3 & $\begin{array}{l}287-441(155 \mathrm{aa}) / \\
859-1323(465 \mathrm{bp} \text {, exon 9-11) }\end{array}$ & NADPH binding & $\begin{array}{l}\text { None } \\
\text { G1236A }\end{array}$ & 11 & New & $\mathrm{E} 412^{\mathrm{h}}$ & $\begin{array}{l}7.25 \\
7.25\end{array}$ \\
\hline 4 & $\begin{array}{l}525-847 \text { (323 aa)/ } \\
1573-2541 \text { (969 bp, exon 13-20) }\end{array}$ & FMN/5-FU binding & $\begin{array}{l}\text { None } \\
\text { G1601A } \\
\text { A1627G } \\
\text { T1896C } \\
\text { In 14 G1Ai } \\
\text { G2194A }\end{array}$ & $\begin{array}{l}13 \\
13 \\
14 \\
14 \\
18\end{array}$ & $\begin{array}{l}* 4 \\
* 5 B \\
- \\
* 2 A \\
* 6\end{array}$ & $\begin{array}{l}\text { S534N } \\
\text { I543V } \\
\text { F632 } \\
\text { exon } 14^{\mathrm{j}} \\
\text { V732I }\end{array}$ & $\begin{array}{l}5.41 \\
5.41 \\
5.41 \\
5.41 \\
6.51 \\
5.41\end{array}$ \\
\hline 5 & $\begin{array}{l}1-26 \text { (26 aa)/1-78 (78 bp, exon 1-2) \& } \\
848-1025(178 \text { aa)/ } \\
2542-3075 \text { (534 bp, exon } 20-23)\end{array}$ & C-terminal Fe-S clusters & $\begin{array}{l}\text { None } \\
\text { A2846T } \\
\text { G2858C }\end{array}$ & $\begin{array}{l}22 \\
22\end{array}$ & New & $\begin{array}{l}\text { D949V } \\
\text { C953S }\end{array}$ & $\begin{array}{l}8.03 \\
8.20 \\
8.08\end{array}$ \\
\hline
\end{tabular}

aAmino acid position; 'base pair numbering based on cDNA sequence: position 1 is $A$ of the start codon ATG; ${ }^{\circ}$ The function of the domain; ${ }^{d}$ SNP, single nucleotide polymorphism, on mRNA; 'Exon containing SNP; fAmino acid substitution on DPD protein; Isoelectric point (pl) of each domain with or without SNP calculated using EMBOSS program; 'Silent mutation; 'The DPYD splicing site mutation; 'deletion of 55 amino acids (residues 581-635).

Table 1: Summary of the human dihydropyrimidine dehydrogenase (DPD) domain structure, function, and SNPs associated with severe 5-FU toxicity (mainly leucopenia and mucositis) reported by the German Clinical Study Group (Appendix Table A7) [2]. With 92\% homology to pig liver DPD (NP_999209.1; see Table 2), the human DPD (NP_000101.2) subunit is also composed of five domains and their distinct functions. 
4. Paste DPD sequence (wild type and mutants) or upload it into each prediction program

\section{Hit prediction}

6. Compare the results or align them side by side (Figure 1)

7. Identify any change in the secondary structural elements

8. If there is, analyze whether or not the change falls within or outside the functional sites of DPD

9. If the change occurs within the functional sites, DPD may be partially or completely dysfunctional

10. Catabolism of 5-FU can be much slower with partial or non DPD activity, resulting in accumulation of 5-FU in the body

11. If DPD is dysfunctional, plasma 5-FU concentration reaches to toxic level with a therapeutic dose

12. Then, a patient with that DPYD mutation is predicted to suffer from adverse side effects

\section{Results}

\section{Amino acid sequence of DPD is evolutionary conserved in phylogenetically related 14 eukaryotes}

To see whether DPD functional sites are evolutionary conserved, phylogenetically related 14 eukaryotes were compared with respect to their amino acid sequence of the DPD monomer. Indeed, the amino acid sequence of DPD, particularly its functional sites in each domain, is well conserved from A. thaliana to human, although there are a few conservative mutations: arginine $(\mathrm{R})$ to lysine $(\mathrm{K})$, threonine (T) to serine (S), or isoleucine (I) to threonine (T) (data not shown). This suggests that a mutation at or near functional sites may result in changes in the secondary structure, and thereby interfere with the DPD function.

\section{Three of eleven SNPs change the secondary structure and function of DPD domains}

It is difficult to draw a conclusion about our body's response to a single drug when multiple drugs are used in combination. As such, the data from toxicity associated with the DPYD genotype in response to 5-FU monotherapy was used to study the consequences of mutational event [2]. Table 1 summarizes the human enzyme DPD structure and function, and its SNPs associated with severe 5-FU toxicity. With 92\% homology to pig liver DPD (Table 2), human DPD monomer can be also divided into five domains with their distinct functions. Twelve out of thirteen SNPs reported (Table A7 from ref. 5) are a point mutation in the coding region, and one SNP in a splicing region. Since E412 in domain 3 (SNP G1236A) and F632 in domain 4 (SNP T1896C) are synonymous mutations, eleven DPYD SNPs are subject to analysis for changes in the domain structure and their impact on the function of DPD using three secondary structure prediction programs, YASPIN and PSIPRED whose predictions most closely match the pig DPD crystal structure, and Jpred 3. As shown in Table 2, the predicted secondary structure of each human DPD domain is $70-88 \%$ identical to the pig DPD crystal structure. The secondary structure and function of each human DPD domain containing point mutations are predicted as follows.

The N-terminal Fe-S clustering domain I (aa 27-173, total 147 aa) with two missense mutations, C29R (SNP T85C) and M166V (SNP A496G) (Table 1). Cysteine 29 (C29) is located within the N-terminal coil next to the first $\alpha$-helix that is not a part of the domain core involved in [4Fe-4S] clusters, and is not one of seven sulfur donors forming two [4Fe-4S] clusters (Figure 1). Furthermore, the predicted secondary structure of domain I with the C29R substitution results in a short extension of the $\mathrm{N}$-terminal coil or no structural change (Figure 1). Besides, C29 is not conserved: human is the only species that has cysteine residue: histidine in pig and zebra fish, arginine in chimpanzee, mouse, rat, and dog or valine in worm and fly (not shown). Although the domain I basicity slightly increases by 0.22 units ( $\mathrm{pI}$ of domain $\mathrm{I}=8.20$ ) (Table 1 ), the $\mathrm{C} 29 \mathrm{R}$ exchange unlikely interrupts the secondary structure of domain I. Similarly, methionine 166 (M166), located at the end of the last $\alpha$-helix of domain I, clearly does not participate in [4Fe-4S] cluster binding. YASPIN and PSIPRED predict the M166V mutation causes little change in the secondary structure of domain I. Therefore, both C29R and M166V mutations have little impact on the domain I function.

The FAD-binding domain II (aa 173-286 and 442-524, total 196 aa) with three missense mutations, R208Q (SNP G623A), K259E (SNP A775G), and V464A (SNP T1391C) (Table 1). First, arginine 208 (R208) is located at the end of $\alpha$-helix followed by a short coil and $\beta$-strand (Figure 1). The R208Q substitution occurs not in the sites where the diphosphate moiety of FAD binds (A198, L226 and D481), and causes little change in the domain II secondary structure (Figure 2 ), in spite of a slight increase in its acidity by 0.15 units ( $\mathrm{pI}$ of domain $\mathrm{I}=4.85$ ) (Table 1). Second, lysine 259 (K259) is located within coil close to L261, one of the binding sites to the adenosine moiety of FAD, which includes E218 and K219. Unexpectedly, a dramatic substitution of a basic to acidic amino acid (K259E) results in little change in the secondary structures of domain II (Figure 1). Finally, valine 464 (V464) is located in coil (or $\beta$-strand). The conservative V464A mutation has no effect on the domain II secondary structure, confirmed by two programs (Figure 1). Taken together, all three substitutions, R208Q, $\mathrm{K} 259 \mathrm{E}$, and V464A, are not involved in the cofactor FAD-interacting sites including its isoalloxazine ring (R235, T489, and V129 in Domain 1 ), and have little change in the secondary structure, indicating that the domain II function is intact with each mutation.

The FMN and 5-FU binding domain IV (aa 525-847, total 323 aa) with four missense mutations, S534N (SNP G1601A), I543V (SNP A1627G), V732I (SNP G2194A) and exon 14 skipping (SNP In14 G1A) (Table 1). Domain IV forms the barrels with $\beta$-strands and $\alpha$-helices containing four cis-peptide bonds. In this slightly acidic domain IV (pI of domain IV=5.41), the three conservative mutations (S534N, I543V, and V732I) are not involved in cis-bond formation. The secondary structure of domain IV with each mutation shows either no change or minor change (Figure 1). Most importantly, S534, I543, and V732 are not the binding sites for FMN or 5-FU (Figure 1). As such, the domain IV function is not interrupted with those mutations. However, a transition from $\mathrm{G}$ to $\mathrm{A}$ at position 1 in exon 14 in a splicing region (allele ${ }^{\star} 2 \mathrm{~A}$ ) skipped entire exon 14 , causing a deletion of 55 amino acids (D581-N635) that include the cis-peptide bond-forming residues (S587 and P588), the 5-FU interacting residue and its surrounding residues (N609 and I613), the residue interacting with the isoalloxazine ring of FMN (K574), and the residues interacting with K574 (E611 and L612). It is obvious that this entire deletion of exon 14 disrupts the domain IV barrel structure as well as ligand binding, subsquently leading to a complete loss of DPD activity.

The C-terminal Fe-S clustering domain V (aa 1-26 and 848-1025, total $204 \mathrm{aa}$,) with two missense mutations, D949V (SNP A2846T) and C953S (SNP G2858C) (Table 1). Aspartic acid 949 (D949) is located 
Citation: Son K (2015) Prediction of 5-Fluorouracil Toxicity Associated With Dihydropyrimidine Dehydrogenase Gene (DPYD) Polymorphism Using the Secondary Structure Prediction Programs. J Pharmacogenomics Pharmacoproteomics 6: 154. doi:10.4172/2153-0645.1000154
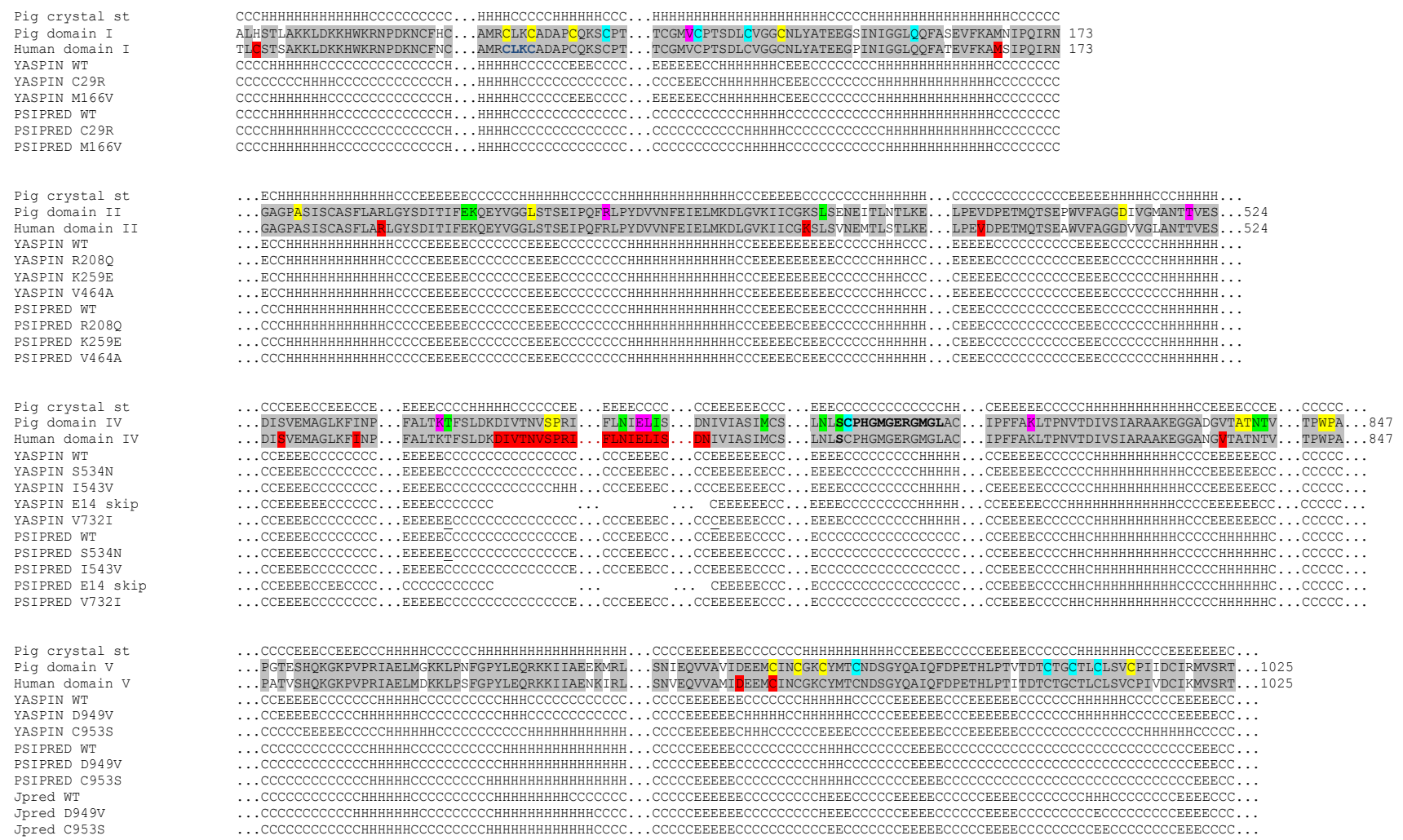

Figure 1: Two missense point mutations, D949V (SNP A2846T) and C953S (SNP G2858C), and one exon 14 deletion (SNP In14 1G>A) change or delete core sites in the secondary structure of the human DPD domains responsible for 5-FU metabolism. Exon 14 skipping caused a deletion of 55 amino acids (D581-N635). The secondary structure prediction programs, YASPIN, PSIPRED and Jpred 3, were used to predict the secondary structures of human DPD domains and their subsequent changes due to SNPs-derived missense point mutations. The secondary structure elements taken from the pig DPD crystallographic analysis are shown for comparison. NADPH-binding domain III sequence is not shown as it contains a synonymous SNP (Table 1). Some of the domain sequences not involved in SNPs or functions are omitted due to the space limitation. Conserved residues between human and pig are shaded in light grey. The amino acids substituted or deleted in human DPD due to SNP including fifty-five amino acids (D581-N635) corresponding to exon 14 highlight in red. Pig DPD functional sites [9] are highlighted with colors as follows. In domain I, C79, C82, C87 and C140 (yellow) and C91, C130, C136 and Q156 (cyan) coordinate two Fe-S clusters. In domain II, E218, K219, and L26 (light green) directly interact with adenosine moiety of FAD; L226, A198, and D481 (yellow) bind to its diphosphate moiety; R235, T489, and V129 in domain 1 (pink) interact with the isoalloxazine ring of FAD. In domain IV, FMN interacts with three cis-peptide bonds (S587 and P588, W751 and P752, A734 and T735, yellow), K574 and K709 (pink) binding to its isoalloxazine ring, and K574-interacting E611 and L612 (pink). 5-FU interacts with N609, N668, N736, T737 and S670 (light green), indirectly with the side chains of M642, I613, T575, and N668 (light green), with the active site loop (S670-L682) (bold letters) including the catalytically crucial C671 (cyan). In domain V, C953, C956, C959 and C996 (yellow) and C963, C986, C989 and C992 (cyan) coordinate two Fe-S clusters. H: Helix, E: Strand, C: Coil.

\begin{tabular}{|c|c|c|c|}
\hline \multicolumn{2}{|c|}{ Human DPD domain (\%) } \\
\hline Pig DPD sequence homology & 93.2 & 92.9 & 94.5 \\
\hline Secondary structure prediction program & $\mathrm{I}$ & II /III & IV \\
\hline YASPIN & 71.4 & 74.6 & 77.1 \\
PSIPHRED & 70.1 & 74.9 & 72.5 \\
\hline
\end{tabular}

Table 2: The alignment scores between the predicted secondary structure of human DPD and the pig DPD crystal structure. The secondary structure prediction programs, YASPIN and PSIPRED, were used to predict the secondary structures of each human DPD domain and compared with the secondary structure taken from the pig DPD crystallographic analysis [9] using the Clustal W program.

closely to cysteine 953 (C953), one of eight metal-binding cysteine residues in the domain core (N940-T1009) formed by two $\alpha$-helices and four $\beta$-strands (Figure 1). YASPIN predicts the core structure closer to the crystal structure compared with PSIPRED, and their predicted secondary structure due to the D949V or C953S substitution differ significantly each other. With the D949V mutation, a short $\alpha$-helix (E950-I954) is generated within coil after a first $\beta$-strand, and the C953S mutation disorganizes the core $\alpha$-helix and $\beta$-strand (YASPIN). On the other hand, little changes in the domain $\mathrm{V}$ secondary structure with both substitutions (PSIPRED), in spite of a slight increase in the domain $\mathrm{V}$ basicity with the D949V substitution (Table 1). Therefore, it is difficult to draw a conclusion from these two prediction programs, and thus the third prediction program, Jpred 3, is introduced to gain a better insight. It is important to point out that, although 2-3 programs are used here to predict the structure-functional relationship, it is in general better to use as many programs as possible to improve prediction. Jpred 3 prediction is closer to the crystal structure compared with PSIPRED, and predicts a different pattern of change, the disruption of a second helix involved in [4Fe-4S] cluster binding (C989 and C992) with the D949V and C953S substitution. Taken together, both D949V and C953S mutation most likely interfere with $[4 \mathrm{Fe}-4 \mathrm{~S}]$ clusters. 
Citation: Son K (2015) Prediction of 5-Fluorouracil Toxicity Associated With Dihydropyrimidine Dehydrogenase Gene (DPYD) Polymorphism Using the Secondary Structure Prediction Programs. J Pharmacogenomics Pharmacoproteomics 6: 154. doi:10.4172/2153-0645.1000154

Page 5 of 6

\section{Exon 14 skipping, D949V and C953S change the three dimensional structure of human DPD domains}

To validate the secondary structure predictions, SWISS-MODEL is used to predict the $3 \mathrm{D}$ structure of pig and human DPD domains as well as human DPD domain with SNPs. Homo dimeric pig and human DPD domain I, II/III and IV with the 92.9 to $94.5 \%$ homology (Table 2) share very similar or identical quaternary structures (Figure 2 ; the domain I 3D image is not shown), while with the $88.2 \%$ homology (Table 2) domain $\mathrm{V}$ look very different in their quaternary structures (Figure 3). K259E mutation in domain II/III has little change in its $3 \mathrm{D}$ structure (Figure $2 \mathrm{~A}$ ), as predicted by the secondary structure prediction (Figure 1). In G1A mutation which deletes 55 amino acids in domain IV changes wild type DPD quaternary structure (Figure 2B), missing loops and $\beta$-sheet (blue and green) responsible for interacting FMN and a substrate 5-FU. As shown in Figure 3, human DPD domain $\mathrm{V}$ in the SWISS-MODEL looks very different from its pig counterpart. Both D949V and C953S mutations in this domain dramatically change their wild type DPD quaternary structure by introducing a new helix (dark blue) adjacent to a stretch of helix (cyan), $\beta$-sheet in a loop (dark blue), a longer loop (green), and a shorter helix (light red) responsible for Fe binding (Figure 3). Interestingly, the introduction of these mutations causes their 3D structure very similar to pig 3D (Figure 3 ).

\section{Discussion}

5-FU metabolizing enzyme DPD is expressed in many cell types throughout our body, while the greatest quantity is present in the liver where many drugs including 5-FU are degraded and eliminated. Since $5-F U$ is rapidly degraded by DPD, its effective dose is usually very high for most of patients. This identical effective dose, however, can be life-threatening to small population of patients who carry a natural point mutation in DPYD, if a missense point mutation changes the DPD secondary structure within its functional domain. As DPD is a cytosolic enzyme, a small change in hydrophobicity of sites not involving its functions due to polymorphisms may not significantly affect its conformation. Indeed, most of SNPs leading to a single amino acid exchange analyzed here are predicted to be little change in the DPD secondary structure and thus little effect on its function, DPD activity to break down 5-FU. However, two single missense mutations, D949V (SNP A2846T) and C953S (SNP G2858C), in the C-terminal Fe-S binding domain $\mathrm{V}$ may cause disruption of the core secondary structures of that domain responsible for $[4 \mathrm{Fe}-4 \mathrm{~S}]$ cluster binding, resulting in a partial or complete loss of DPD activity. SWISSMODEL also shows significant changes in the quaternary structure of homo-dimeric DPD domain V with D949V and C953S mutation. Moreover, In14 1G>A mutation missing 55 amino acids (D581-N635) due to an entire deletion of exon 14 is predicted to be a complete loss of DPD activity, which is supported by a significant loss of loops and $\beta$-sheet interacting with FMN and 5-FU from the 3D structure of DPD domain IV (Figure 2 and 3). This prediction is in good agreement with the 5-FU clinical study that Schwab et al reported [2]. Four patients carried the defective $D P Y D^{\star} 2 A$ allele, among 683 patients, developed Grade 4 toxicity, mainly severe mucositis and leucopenia (0.59\%), a population of patients that shows a good correlation between SNP and DPD activity. Therefore, these secondary structure prediction programs can predict structure-associated disruption of DPD function, and can be used to predict SNP-associated toxicity. From a prediction point of view, In14 1G $>$ A (DPYD ${ }^{\star} 2 A$ allele), D949V and C953S are the important genotypes for pharmacogenomic syndrome among 11 SNPs tested here.
With an individual's DPYD genotype in hands following the FDA's recommendation [11], health professionals are able to find out an occurrence of mutation (s) by aligning a patient's DPYD sequence with respect to a DPYD reference sequence. If there is a mutation, then an amino acid substitution or deletion due to a single base mutation can be easily identified. Using the secondary structure prediction programs, they can compare two structures, one from a patient and the other from a normal individual, side by side to examine any change in the secondary structure and whether the change occurs in functional sites in one of five DPD domains. If the structural change falls in a core site of a functional domain, clinicians should check that patient's DPD activity in blood to verify and validate the prediction. If a patient is indeed partially or fully deficient in DPD, they should try to make the best possible intervention unique to that patient. If DPD-deficient patient has no other choice than 5-FU therapy, effective 5-FU doses must be adjusted according to a patient's pharmacokinetic profile and DPD activity in order to maintain efficacy and reduce the toxicity of 5-FU $[12,13]$. Once treatment starts, 5-FU alone or in combination with other drugs such as cisplatin, routine monitoring of DPD activity is required [14].

In conclusion, prediction by these secondary structure programs provides useful and reliable information about changes in the DPD secondary structure and helps determine whether the change occurs

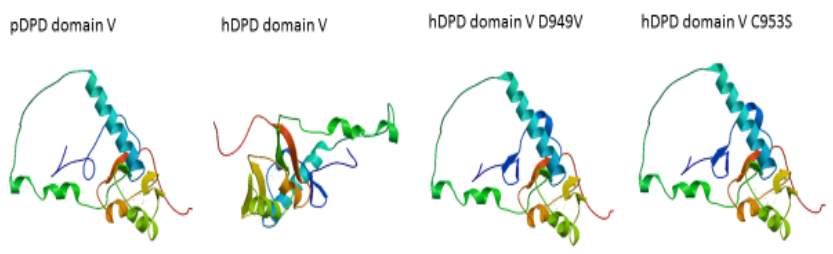

Figure 2: The effect of a point mutation on the three dimensional (3D) structures of the human homo-dimeric DPD domain II/III (A) and domain IV (B). A DPD domain II/III sequence of pig (top left), human (top middle) and its mutant K259E (top right) (A) and the DPD domain IV sequence of pig (bottom right), human (bottom middle), and its mutant exon 14 skipping (In G1A, bottom right) (B) were subjected to the SWISS-MODEL to predict their quaternary structures. Exon 14 skipping, but not K259E mutation significantly changes core functional sites of the DPD domain IV 3D structure.

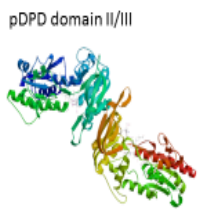

B

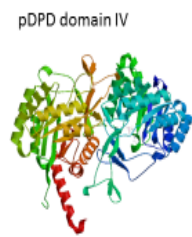

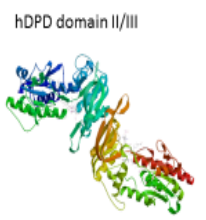

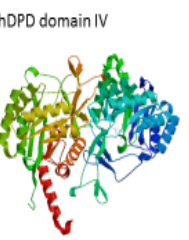

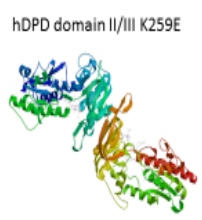

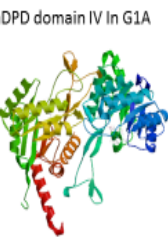

Figure 3: The effect of a point mutation on the three dimensional (3D) structures of the homo-dimeric human DPD domain $\mathrm{V}$. A DPD domain $\mathrm{V}$ sequence of pig (left), human (second left) and its point mutants, D949V (second right) and C953S (right) was subjected to the SWISS-MODEL to predict their quaternary structures. Both mutants alter the 3D structure of DPD domain $V$ to interfere Fe-S clustering. 
Citation: Son K (2015) Prediction of 5-Fluorouracil Toxicity Associated With Dihydropyrimidine Dehydrogenase Gene (DPYD) Polymorphism Using the Secondary Structure Prediction Programs. J Pharmacogenomics Pharmacoproteomics 6: 154. doi:10.4172/2153-0645.1000154

in or outside functional sites of DPD domains. These prediction programs are able to predict whether a point mutation such as In 14 G1A, A2846T, and G2858C disrupts 5-FU metabolism partially or completely. Not only reported SNPs other than 11 point mutations tested here but also a newly discovered SNP can be analyzed by using these prediction programs to predict a patient's tolerance/intolerance to 5-FU. These prediction programs are simple, easy and fast to use, do not require mathematical computing knowledge nor patient's blood testing.

\section{References}

1. Wigmore PM, Mustafa S, El-Beltagy M, Lyons L, Umka J, et al. (2010) Effects of 5-FU. Adv Exp Med Biol 678:157-64.

2. Schwab M, Zanger UM, Marx C, Schaeffeler E, Klein K, et al. (2008) A Role of genetic and non-genetic factors for fluorouracil treatment-related severe toxicity: A Prospective clinical trial by the German 5-Fluorouracil Toxicity Study Group. J Clin Oncol 26: 2131-2138.

3. Saif MW (2013) Dihydropyrimidine dehydrogenase gene (DPYD) polymorphism among Caucasian and non-Caucasian patients with 5-FU-and capecitabinerelated toxicity using full sequencing of $D P Y D$. Cancer Genomics Proteomics 10: 89-92.

4. van Kuilenburg $A B$, Häusler $P$, Schalhorn $A$, Tanck MW, Proost $J H$, et al (2012) Evaluation of 5-fluorouracil pharmacokinetics in cancer patients with a c. $1905+1 \mathrm{G}>$ A mutation in DPYD by means of a Bayesian limited sampling strategy. Clin Pharmacokinet 51:163-174.

5. Rice P, Longden I, Bleasby A (2000) EMBOSS: The European molecular biology open software suite. Trends Genet 16: 276-277.
6. Lin K, Simossis VA, Taylor WR, Heringaa J (2005) A simple and fast secondary structure prediction algorithm using hidden neural networks. Bioinformatics 21 : 152-159.

7. Buchan DWA, Minneci F, Nugent TCO, Bryson K, Jones DT (2013) Scalable web services for the PSIPRED protein analysis workbench. Nucl Acids Res 41 W340-W348.

8. Cole C, Barber JD, Barton GJ (2008) The Jpred 3 secondary structure prediction server. Nucl Acids Res 36: W197-W201.

9. Dobritzsch D, Schneider G, Schnackerz KD, Lindqvist Y (2001) Crysta structure of Dihydropyrimidine Dehydrogenase, a major determinant of the pharmacokinetics of the anticancer drug 5-fluorouracil. EMBO J 20: 650-660.

10. Arnold K, Bordoli L, Kopp J, Schwede T (2006) The SWISS-MODEL Workspace: A web-based environment for protein structure homology modelling. Bioinformatics 22: 195-201.

11. www.fda.gov

12. Bocci G, Barbara C, Vannozzi F, Di Paolo A, Melosi A, et al. (2006) A pharmacokinetic-based test to prevent severe 5-fluorouracil toxicity. Clin Pharmcol Ther 80: 384-395.

13. van Staveren MC, Jan Guchelaar $H$, van Kuilenburg ABP, Gelderblom H, Maring JG (2013) Evaluation of predictive tests for screening for dihydropyrimidine dehydrogenase deficiency. Pharmacogenomics J 13: 389-395.

14. Magne N, Pivot X, Etienne-Grimaldi MC, Francois E, Renee N, et al. (2003) Impact of platinum complexes on dihydropyrimidine dehydrogenase activity in 5-fluorouracil-treated patients. Ann Incol 14: 1329-1330. 